\title{
Effect of transvection on the expression of the Notch locus in Drosophila melanogaster
}

\author{
Mariitta Sirén and \\ Petter Portin
}

Laboratory of Genetics, Department of Biology, University of Turku, SF-20500 Turku 50, Finland.

The effect of different $X$ chromosomal inversions in a heterozygous condition on the expression of the recessive lethal Abruptex mutations of the Notch locus of Drosophila melanogaster was studied by measuring quantitatively the effect of Abruptex on the number of bristles, and on wing venation. The Abruptex mutations were combined in cis with recessive mutations located in the same exon, on the one hand, and in the large intron of the locus on the other. All the inversions studied caused some suppression of the mutant bristle phenotype, and the complex inversions a suppression of the wing venation phenotype, as well. This is consistent with the hypothesis that transvection causes a reduction in the amount of the gene product, since lethal Abruptex mutations are antimorphic mutations. One short inversion caused an enhancement of the wing venation phenotype. Compounds of exon and intron mutations in cis gave similar results, which are discussed from the point of view of the enhancer hypothesis of transvection. In general, the results suggest that transvection is a general phenomenon in Drosophila melanogaster, possibly involving all loci.

\section{INTRODUCTION}

The effect of structural heterozygosity on the expression of genes on homologous chromosomes was discovered and termed transvection by Lewis (1954) in the bithorax complex locus of Drosophila melanogaster. In the bithorax locus transvection induced an enhancement of the mutant phenotype of alleles in the trans position, and was thought to be due to the prevention of the opposition of homologous alleles (Lewis, 1954; Gelbart, 1982). In addition to the effect at the bithorax complex (BX-C; map location 3-58.8) transvection has been observed in the decapentaplegic complex (DPP-C; 2-4.0) (Gelbart, 1982) and at the white locus $(w ; 1-1 \cdot 5)$ (Green, 1967; Gelbart, 1971, 1982; Jack and Judd, 1979; Gelbart and Wu, 1982; Zachar et al., 1985; Smolik-Utlaut and Gelbart, 1987). Synapsis-dependent trans regulation of gene activity has also been suggested for the salivary gland glue protein $4(S g s-4)$ gene by Korge (1981).

The aim of the present study was to demonstrate the transvection effect at the Notch $(N$; $1-3 \cdot 0$ ) locus of $D$. melanogaster, and further to demonstrate that mutations coupled in cis with the Abruptex $(A x)$ mutations located in the large exon of the locus give similar results irrespective of whether they are located in the same exon (split, $s p l$ ) or in a distally located large intron (facetglossy, $\left.f a^{g}\right)$. We have previously demonstrated that these mutations have an effect on Abruptex, split in general enhancing and facet-glossy suppressing the expression of Abruptex in cis-compounds (Sirén and Portin, 1984). This result will be discussed in the light of the enhancer hypothesis of the transvection effect of Zachar et al. (1985). In general our results support the enhancer hypothesis, since they suggest that transvection is a general phenomenon in $D$. melanogaster, possibly involving all loci. Also, the results support the hypothesis that the transvection effect is due to a reduction in the amount of the gene product from the structurally normal homologue.

\section{MATERIALS AND METHODS}

The Abruptex $(A x, 1-3 \cdot 0)$ mutations of the Notch locus make it possible to measure gene expression quantitatively since they cause missing bristles on the head and thorax, and breaks in the wing veins. We counted the mean numbers of missing orbital, dorsocentral, and scutellar bristles; counted the 
number of wing breaks, and measured their length microscopically. The Abruptex mutations used were $A x^{59 b 8 \cdot 1}$ and $A x^{59 d 5}$ which are recessive lethal mutations (Welshons 1971), and antimorphic in their mode of action (Portin, 1981). The expression of these $A x$ mutations was measured in females heterozygous for either a normal $\mathrm{X}$ chromosome or three different structurally mutant $X$ chromosomes. The latter ones were $\operatorname{In}(1) F M 6, B a s c$, and In(1) Lü-2. Information on the complex rearrangements in In(1)FM6 and Basc can be found in Lindsley and Grell (1968). In(1)Lü-2 was kindly provided by Professor K. G. Lüning (Stockholm, Sweden), and is an inversion of 50 bands between the loci of white $(w, 1-1.5)$ and curlex $(c x, 1-13 \cdot 6)$ (Lüning, personal communication). The $A x$ mutations were investigated in simple heterozygotes, or they were coupled with either of two different visible mutations of the Notch locus, namely split $(s p l)$ and facet-glossy $\left(f a^{g}\right)$.

The molecular fine structure map of the Notch locus is presented in fig. 1. In the figure the locations of $f a^{g}$ and $s p l$ are given as well as the approximate locations of $A x^{59 b 8.1}$ and $A x^{59 d 5} \cdot f a^{g}$ is a short insertion in the large intron of the locus (Artavanis-Tsakonas et al., 1984; Kidd and Young, 1986), while $s p l$ is a point mutation in the large exon of the locus (Hartley et al., 1987). The

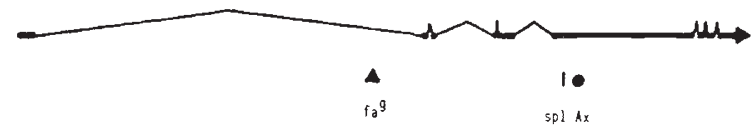

Figure 1 The molecular fine structure map of the Notch locus indicating the positions of $f a^{g}$ and $s p l$, and the approximate positions of $A x^{59 b 8 \cdot 1}$ and $A x^{59 d 5}$ (designated $A x$ ). Thin lines are intronx, and heavy lines are exons. $f a^{g}$ is an insertion while $s p l$ as well as $A x$ mutations map as a points. Data from Artavanis-Tsakonas et al., 1984; Hartley et al., 1987; Kelley et al., 1987; Kidd et al., 1983; Kidd and Young, 1984.

molecular nature of $A x^{59 b 8.1}$ and $A x^{59 d 5}$ as well as of five viable $A x$ mutations has been indicated quite recently. They map as points, and are associated with single amino acid substitutions in the Epidermal Growth Factor-like sequences of the gene product (Kelley et al., 1987).

Flies were reared on a standard Drosophila medium containing semolina, agar-agar, syrup, and both dried and fresh yeast at $25^{\circ} \mathrm{C}$.

The results were statistically analysed by the analysis of variance.

\section{RESULTS}

The expression of the Abruptex phenotype in the different genotypes tested is given in table 1 . All

Table 1 The expression of Abruptex phenotype of different genotypes of the Notch locus involving Abruptex mutations in different combinations with split and facet-glossy mutations and heterozygous with three different $\mathrm{X}$ chromosomal inversions

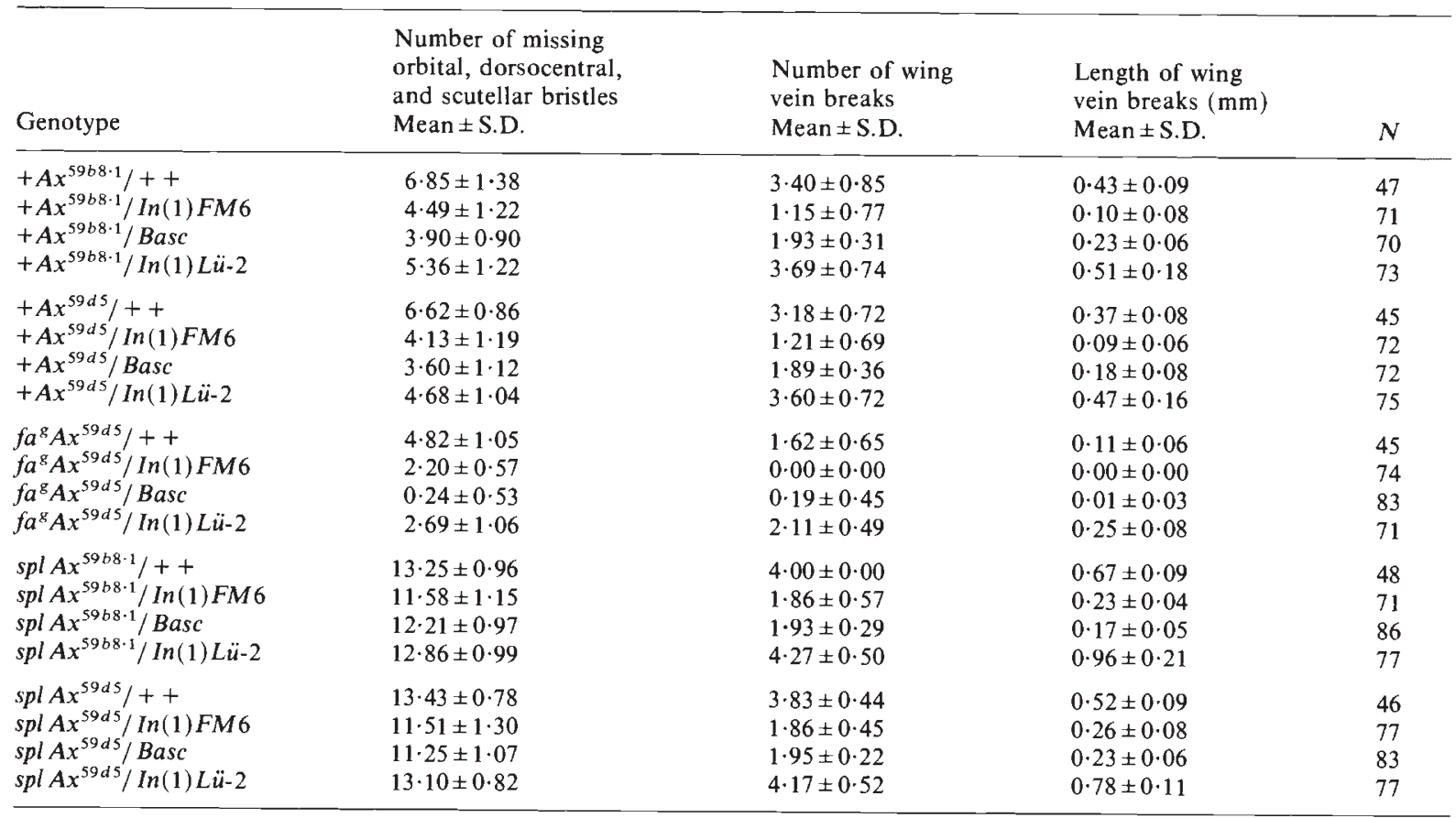


inversions tested caused some suppression of the mutant bristle phenotype in heterozygous condition with all the Notch compounds tested. The complex X chromosomal inversions (In(1)FM6 and $B$ asc) also caused a suppression of the mutant wing venation phenotype in all genotypes tested, while $\operatorname{In}(1) L \ddot{u}$ - 2 caused an enhancement of the wing venation phenotype. This latter exceptional observation is probably due to the vicinity of the curlex $(c x)$ gene of the proximal break point of In(1) $L \ddot{u}-2$. Curlex affects the development of the wings (see Lindsley and Grell, 1968). All differences were statically significant. In general, it may be concluded that structural heterozygosity overlapping the Notch locus in the $\mathrm{X}$ chromosome causes a suppression of expression of Abruptex alleles of the locus.

\section{DISCUSSION}

It was observed that structural heterozygosity in the $\mathrm{X}$ chromosome in general suppresses the expression of the Abruptex mutations of the Notch locus. In the bithorax complex $(B X-C)$ (Lewis, 1954), and in decapentaplegic complex (DPP-C) (Gelbart, 1982) it has been observed that transvection effects enhance the expression of mutant genes. This apparent discrepancy is most probable due to the fact that Abruptex mutations studied are antimorphic (Portin, 1981), while mutations tested for transvection in the $B X-C$ and $D P P-C$ systems are most likely amorphic or hypomorphic mutations. An antimorphic mode of action of the Abruptex mutations was assumed on the basis that an increasing dose of the mutant alleles enhanced; while an increasing dose of the wild type allele suppressed the expression of the Abruptex phenotype.

Thus, the results in all the three systems tested suggest that the transvection effect is due to a reduction in the amount of the mutant gene product from the structurally normal homologue (cf. Micol and Garcia-Bellido, 1987).

Secondly, it is interesting that an intron mutant $\left(f a^{g}\right)$ and an exon mutant $(s p l)$ coupled in cis with Abruptex gave similar results. Zachar et al. (1985) proposed an enhancer hypothesis as a molecular explanation of the transvection effect. They based their suggestion on the possibility that an putative enhancer element of the white locus, $w^{s p}$, activated white transcription in trans in the case of somatically synapsed white genes, and that the white transvection effect potentiating the zeste-white interaction involved the $w^{s p}$ element. Furthermore,
Zachar et al. (1985) suggested that the $w^{s p}$ element activates transcription from the $w^{D Z L}$ transposon unique segment promoter.

Like $w^{D Z L}, f a^{g}$ is also an insertion, although in this case inside the locus in an intron, while spl is a point mutation in the same exon where $A x$ is located. Earlier we have observed that in general $\mathrm{fa}^{\mathrm{g}}$ suppresses and $\mathrm{spl}$ enhances the expression of Abruptex in cis compounds (Sirén and Portin, 1984) (see also table 1). Despite this, they have a similar behaviour in the transvection effect. Therefore, our results might be in contradiction to the details of the enhancer hypothesis of transvection effect.

On the other hand, the enhancer hypothesis of the transvection effect predicts that transvection effects would be quite common. We have observed the transvection effect in the Notch locus which is a single transcroption unit (Kidd et al., 1983; Artavanis-Tsakonas et al., 1983). Thus, the transvection effect is not a property of complex loci like $B X-C$ and $D P P-C$, but might be a general phenomenon in $D$. melanogaster possibly involving all loci. Only the difficulty of quantitative analysis of the gene expression with formal genetic methods makes it difficult to observe transvection effects.

Acknowledgements This work has been supported by the Academy of Finland. We are grateful to Professors K. G. Lüning (Stockholm, Sweden) and W. J. Welshons (Ames, Iowa) for providing us with Drosophila stocks.

\section{REFERENCES}

ARTAVANIS-TSAKONAS, S., GRIMWADE, B. G., HARRISON, R. G., MARKOPOULOU, K., MUSKAVITCH, M. A. T., SCHLESINGER-BRYANT, R., WHARTON, K. AND YEDVOBNICK, B. 1984. The Notch locus of Drosophila melanogaster: A molecular analysis. Developm. Genet. 4, 233-254.

ARTAVANIS-TSAKONAS, S., MUSKAVITCH, M. A. T. AND YEDVOBNICK, B. 1983. Molecular cloning of Notch, a locus affecting neurogenesis in Drosophila melangaster. Proc. Natl. Acad. Sci. USA, 80, 1977-1981.

GELBART, W. M. 1971. Cytogenetics of zeste expression in Drosophila melanogaster. Ph.D. Thesis, University of Wisconsin, Madison, Wisconsin.

GELBART, w. M. 1982. Synapsis dependent allelic complementation at the decapentaplegic complex in Drosophila melanogaster. Proc. Natl. Acad. Sci. USA, 79, 2636-2640.

GELBART, W. M. AND WU, C. T. 1982. Interactions of zeste mutations with loci exhibiting transvection effects in Drosophila melanogaster. Genetics 102, 179-189.

GREEN, M. M. 1967. Variegation of the eye color mutant zeste as a function of a rearrangements at the white locus in Drosophila melanogaster. Biol. Zentralbl., 86, (Suppl), 211 220. 
HARTLEY, D. A., XU, T. AND ARTAVANIS-TSAKONAS, S. 1987 The embryonic expression of the Notch locus of Drosophila melanogaster and the implications of points mutations in the extracellular EGF-like domain of the predicted protein. EMBO J., 6, 3707-3718.

JACK, J. W. AND JUDD, B. H. 1979. Allelic pairing and gene regulation: a model for the zeste-white interaction in Drosophila melanogaster. Proc. Natl. Acad. Sci. USA, 76, 1368-1372.

KELLEY, M. R., KIDD, S., DEUTSCH, W. A. AND YOUNG, M. W. 1987. Mutations altering the structure of Epidermal Growth Factor-like coding sequences at the Drosophila Notch locus. Cell, 51, 539-578.

KIDD, S., LOCKETT, T. J. AND YOUNG, M. W. 1983. The Notch locus of Drosphila melanogaster. Cell, 34, 421-433.

KIDD, S. AND YOUNG, M. W. 1986. Transposon-dependent mutant phenotypes at the Notch locus of Drosophila. Nature, 232, 89-91.

KORGE, G. 1981. Genetic analysis of the larval secretion gene Sgs-4 and its regulatory chromosome sites in Drosophila melanogaster. Chromosoma, 84, 373-390.

LEWIS, E. B. 1954. The theory and application of a new method of detecting chromosomal rearrangements in Drosophila melanogaster. Am. Nat. 88, 225-239.
LINDSLEY, D. L. AND GRELL, E. H. 1968. Genetic Variations of Drosophila melanogaster. Carnegie Institution, Washington, D. C.

MICOL, J. L. AND GARCIA-BELLIDO, A. 1987. Genetic analysis of transvection effects involving Contrabithorax mutations. 10th Eur. Dros. Res. Conf. Abstr. 176.

PORTIN, P. 1981. The antimorphic mode of action of lethal Abruptex alleles of the Notch locus in Drosophila melanogaster. Hereditas, 95, 247-251.

SIRÉN, M. AND PORTIN, P. 1984. Cis-trans tests at the Notch locus of Drosophila melanogaster suggesting multiple cis-acting control sites inside the locus. Hereditas, 101, 49-56.

SMOLIK-UTLAUT, S. M. AND GELBART, W. M. 1987. The effects of chromosomal rearrangements on the zeste-white interaction in Drosophila melanogaster. Genetics, 116, 285-298.

WELSHONS, W. J. 1971. Genetic basis for two types of recessive lethality at the Notch locus of Drosphila. Genetics, 68, 259-268.

ZACHAR, Z., CHAPMAN, C. H. AND BINGHAM, P. M. 1985. On the molecular basis of transvection effects and the regulation of transcription. Cold Spring Harbour Symp. Quant. Biol., 50, 337-346. 